\title{
Megaureter Associated with Severe Renal Dysplasia
}

\author{
Anthony L. Alcantara, MD,* Gary M. Amundson, MD, ${ }^{*}$ \\ and Chung-Ho Chang, MD $\dagger$
}

The diagnosis of megaureter can usually be made quite easily with ultrasonography. A large anechoic tubular structure can be seen with communication to the kidney and/or bladder demonstrated. We present a case in which massive megaureter was found in association with severe renal dysplasia. Inability to demonstrate a connection of the megaureter to the rest of the urinary system caused difficulties in accurate radiologic diagnosis.

\section{CASE REPORT}

A black female infant, born with an abdominal mass, was examined utilizing multiple modalities to determine the nature of the mass. An ultrasound examination at 4 days of age revealed a cystic mass in the left lower abdomen that appeared to fold upon itself as it extended into the left pelvis and slightly across the midline (Figure 1). Communication between the mass and an abdominal organ could not be demonstrated, although the mass was contiguous with the bladder, which was displaced anteriorly. In addition, a kidney was not seen in the left renal fossa. There was no reflux demonstrated on a voiding cystourethrogram, and renal scintigraphy did not identify a functioning (or ectopic) left kidney. The patient was not seen again in the radiology department until she was 17 months of age, at which time ultrasonography again demonstrated the cystic, apparently septated mass, which had enlarged and extended to both sides of the pelvis. This was thought to be consistent with a large mesenteric cyst (Figure 2).

From the *Department of Radiology, Children's Hospital of Michigan, Detroit, Michigan, affiliated with the Wayne State University School of Medicine, and †Department of Pathology, William Beaumont Hospital, Royal Oak, Michigan. For reprints contact G.M. Amundson, MD, Department of Radiology, Children's Hospital of Michigan, 3901 Beaubien Boulevard, Detroit, Michigan 48201.
Two weeks after the repeat ultrasound examination, the patient presented with a 3 -day history of poor oral intake and progressive emesis. Bowel obstruction secondary to the abdominal mass was suspected and emergent surgery performed. A cystic U-shaped mass was identified, with its open end pointing into the pelvis (Figure 3 ). Further dissection revealed it to be a massive hydroureter. Proximal to this dilated segment, there was an abrupt kink in the ureter, then a 4 $\mathrm{cm}$ - to 5-cm segment of normal size leading to a small dysplastic kidney located in the left renal fossa. An abrupt kink was also present at the distal end of the hydroureter, followed by a more normal-sized segment that had a normally placed orifice in the bladder.

Pathologic examination found a small dysplastic kidney with a small segment of welldeveloped cortex and medulla at one pole (Figure 4). The pelvicalyceal system was poorly formed. The kidney was connected to $5.0 \mathrm{~cm}$ of ureter with a normal diameter but that had its lumen obliterated by abrupt kinking at its junction with the dilated portion of the ureter. The dilated segment of ureter was lined by transitional epithelium, and contained uniformly thickened and hypertrophied muscle layers accompanied by hypertrophied nerve bundles in the serosa. A small opening was present at the distal end of the hydroureter. The findings were consistent with a closed-loop (or sausage-shaped) megaureter.

\section{DISCUSSION}

Megaureter has been classified into three major categories. ${ }^{1,2}$ Type $A$ is ureteral dilatation secondary to vesicoureteral reflux. In Type $B$, ureteral dilatation results from an anatomic obstruction. Type $\mathrm{C}$ is a nonrefluxing, nonobstructed megaureter. Differentiation of Types B and C may be difficult, but is important because there is a differ- 
MEGAURETER WITH RENAL DYSPLASIA

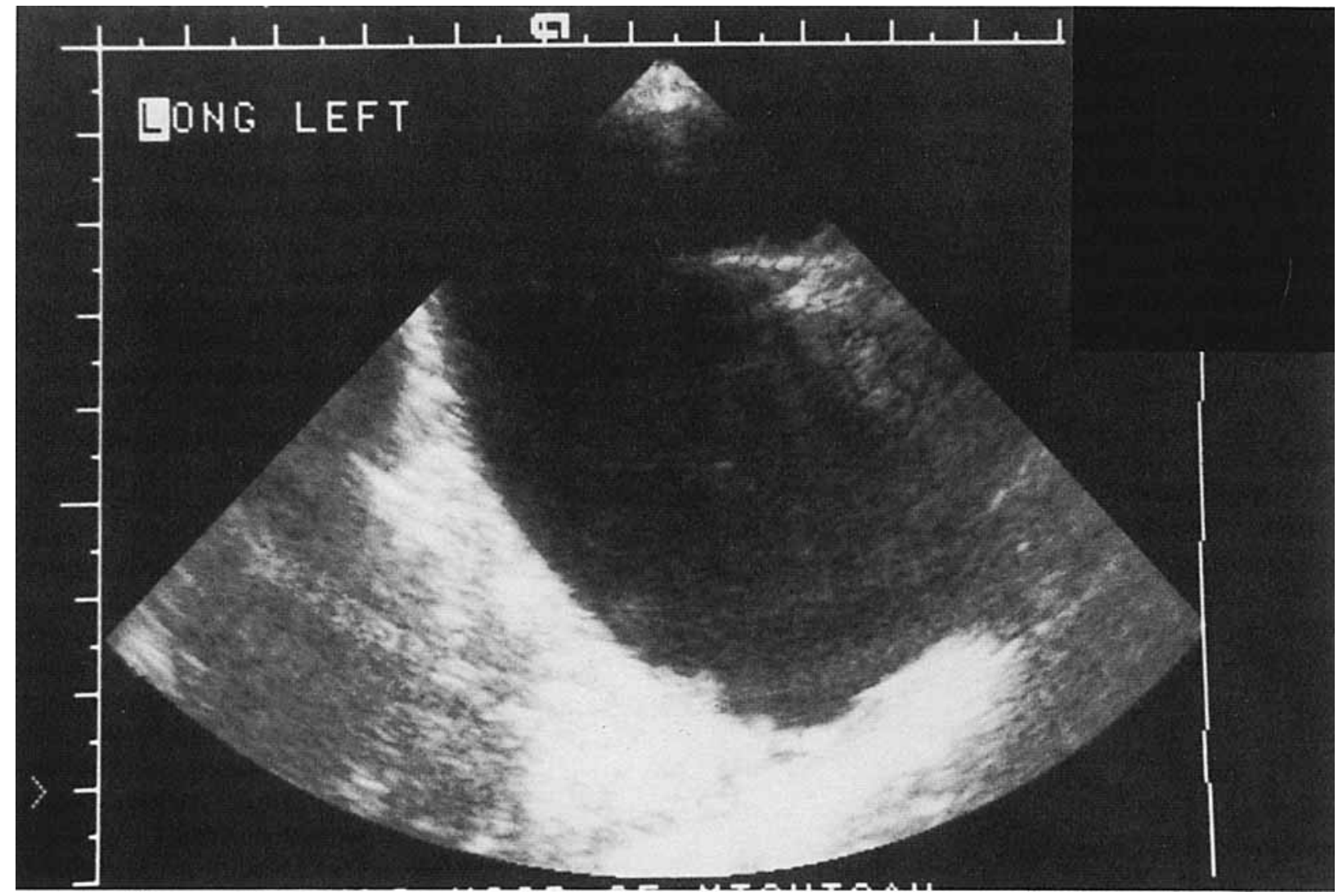

FIGURE 1. Ultrasound scan at 4 days of age. Coronal view of the abdomen from the left flank: a large cystic mass, somewhat tubular but appearing to fold upon itself, is seen in the left lower abdominal quadrant.

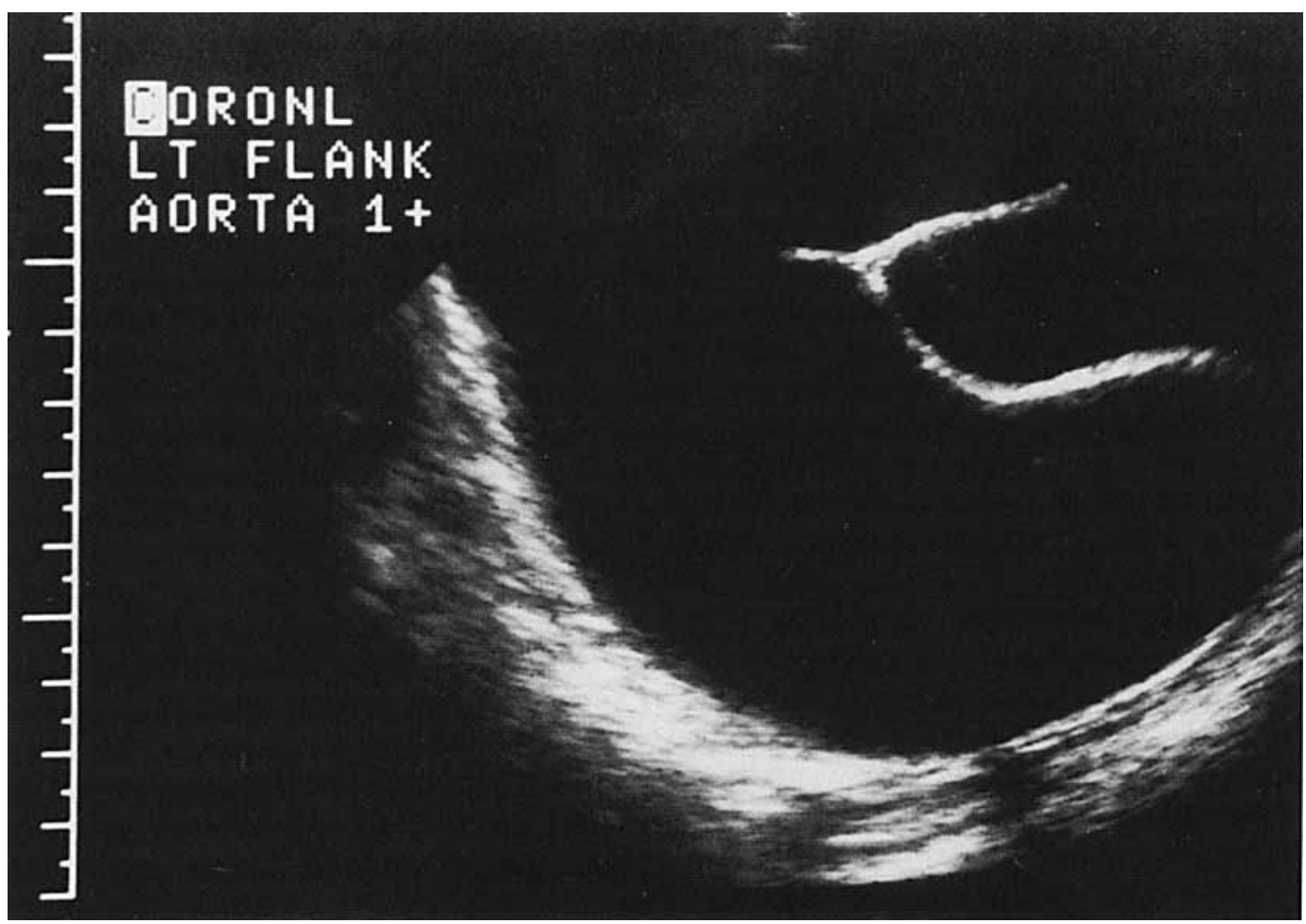

FIGURE 2. Follow-up ultrasound scan at 17 months. Coronal view of the abdomen from the left flank reveals enlargement of the cystic mass with apparent septations. No internal echoes are seen. 


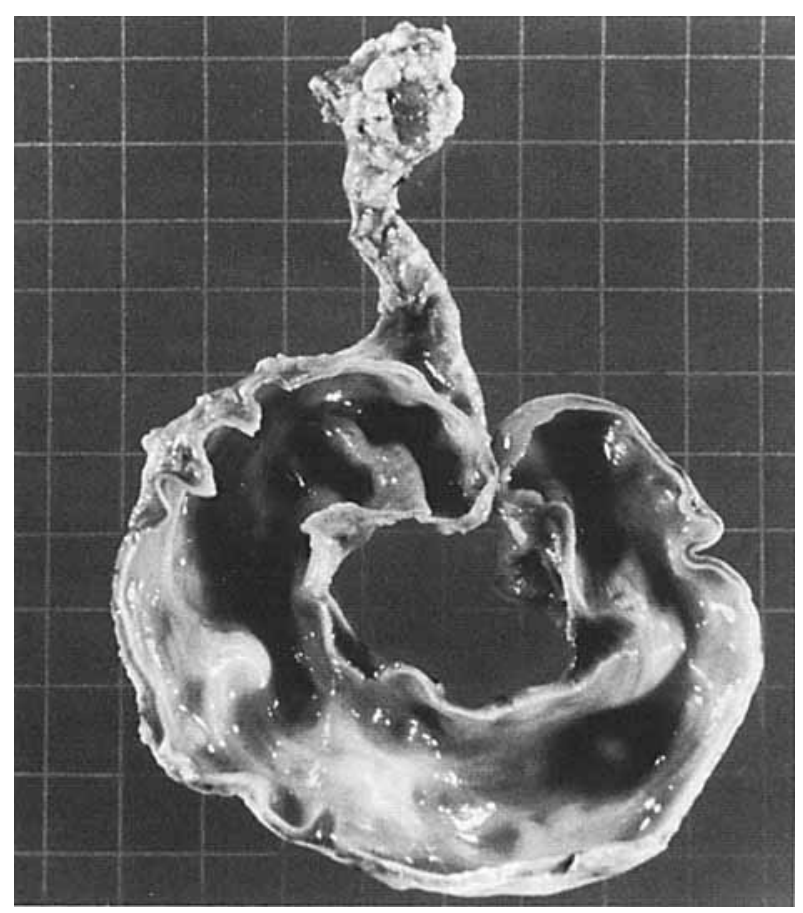

FIGURE 3. Gross pathologic specimen shows a horseshoe-shaped, dilated structure with a small dysplastic kidney attached to one end by a short segment of ureter of normal size. ence in their management. ${ }^{1}$ The pathophysiology of Type $\mathrm{C}$ megaureter remains unclear but several explanations have been proposed. One theory considers the distal narrowed segment to be normal and the proximal segment to be abnormal, with poor peristalsis resulting in dilatation. ${ }^{2}$ Others have postulated that an aganglionic, aperistaltic distal segment results in proximal dilatation, a process analogous to that seen in Hirschsprung's disease of the colon. ${ }^{3}$ Ureteral dilatation due to vesicoureteral reflux that has subsequently ceased has also been considered. ${ }^{2}$ Microscopic examination of nonobstructed megaureters has shown increased wall thickness and muscle cell hypertrophy in the dilated portion. On electron microscopy, a dysplastic appearance may be seen in the hypertrophitd portions with reduction in muscle cells and increased collagen deposition. 4

Megaureter usually results in a variable degree of pyelocaliectasis depending on the degree of the obstruction. An association of primary megaureter with contralateral renal dysplasia is known. ${ }^{5,6}$ Cystic dysplastic kidneys have been seen with megaureters in cases of distal obstruc-

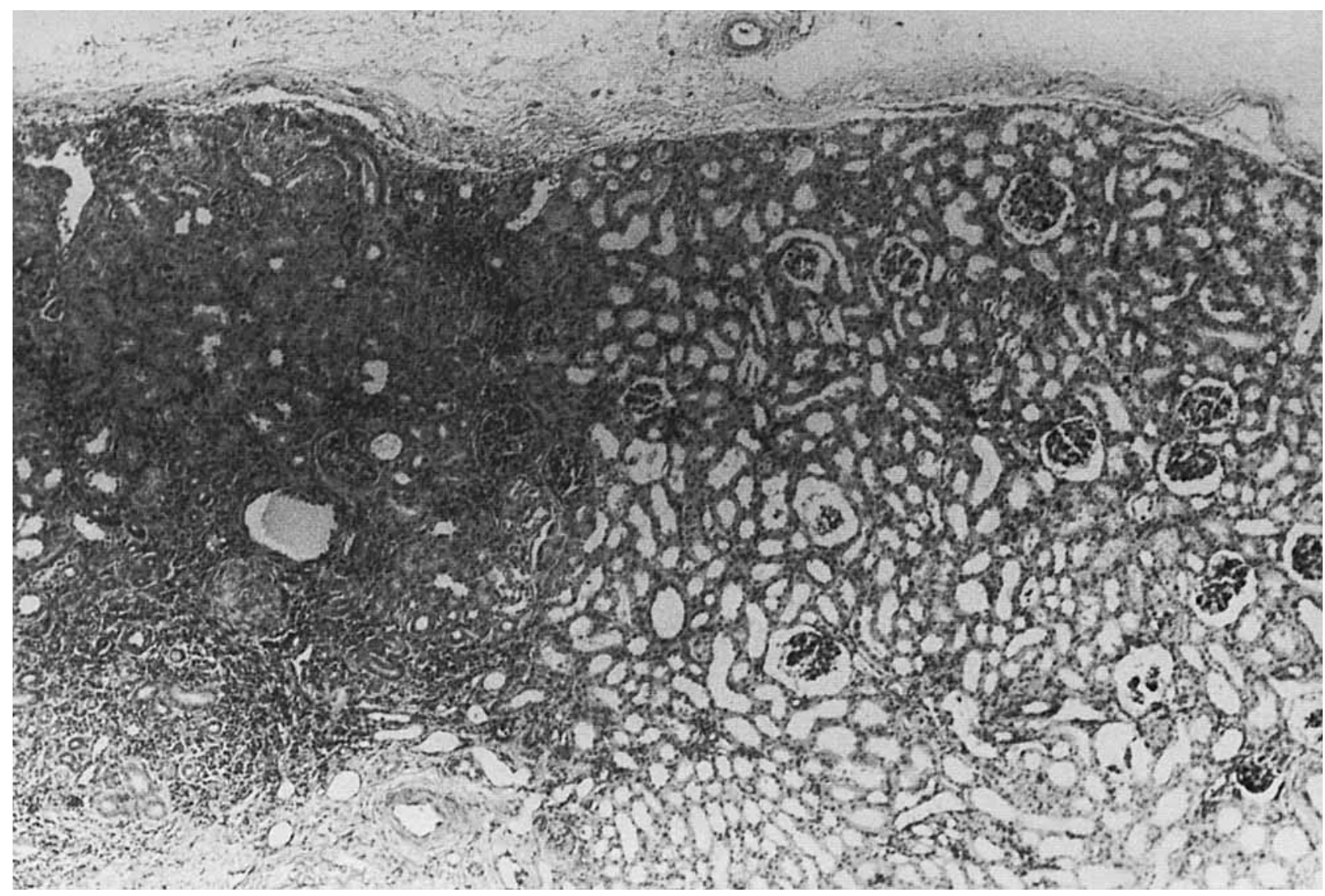

FIGURE 4. Photomicrograph of the part of the kidney showing preservation of the cortex. Note the normal-appearing glomeruli in their usual distribution (Hematoxylin \& Eosin stain, original magnification $\times 40$ ). 
tion. ${ }^{6,7}$ Reports of primary megaureter with severe ipsilateral renal dysplasia are less common. ${ }^{8,9} \mathrm{~A}$ high incidence of ipsilateral renal dysmorphism has been reported when there was extensive muscle dysplasia in megaureters. ${ }^{5}$ In this patient, the hypertrophied segment did not show muscle dysplasia. The severe renal dysplasia is similar to that seen with long-standing obstruction, although dysplasia resulting as a primary or secondary event is generally indistinguishable pathologically. However, the presence of well-developed renal parenchyma without cystic change in a small portion of the kidney suggests that there was function without obstruction. The pathogenesis of the closed-loop megaureter in this case is presumably due to gradual enlargement of the megaureter with eventual twisting or kinking at either end of the dilated segment, resulting in acute abdominal symptoms.

Ureteral dilatation can be recognized sonographically by its tubular appearance and anatomic course. However, when primary megaureter is associated with a severely dysplastic nonvisualized kidney, demonstration that the two are related may be difficult. This was particularly true in this case; the hydroureter became so enlarged and folded upon itself that it was no longer tubular but appeared as a cystic, partially septated mass. Correlation with the pathologic specimen clearly demonstrated that what was shown sonographically as a rounded mass was, in fact, the ureter. Awareness of this rare combination and its sonographic appearance may aid in providing a more accurate diagnosis.

\section{REFERENCES}

1. Keating MA, Retik AB: Management of the dilated obstructed ureter. Urol Clin North Am 17:2, 1990.

2. Stephens FD, Cook WA: Congenital urologic abnormalities, in: Schirer RW, Gottschalk CW (eds): Diseases of the Kidney (ed 4) New York, Little, Brown, 1988, pp 697-702.

3. Swenson O, Fisher JH: The relation of megacolon and megaloureter. New Engl J Med 253:1147, 1955.

4. Hanna MK, Jeffs RD, Sturgess JM, et al: Ureteral structure and ultrastructure. Part III. The congenitally dilated ureter (megaureter). J Urol 117:24, 1977.

5. Pfister RC, Hendren WH: Primary megaureter in children and adults. Clinical and pathophysiologic features of 150 ureters. Urology 12:160, 1978.

6. Brown T, Mandell J, Lebowitz RL: Neonatal hydronephrosis in the era of sonography. AJR 148:959, 1987.

7. Slater GS: Ureteral atresia producing giant hydroureter. J Urol 78:135, 1957.

8. Huang C: Congenital giant megaureter. J Ped Surg 22:235, 1987.

9. Tokunaka S, Gotoh T, Koyanagi T, et al: Muscle dysplasia in megaureters. $J$ Urol 131:383, 1984. 